\title{
Simultaneous Small Intestinal Bleeding and Stricture Caused by Meckel's Diverticulum
}

Shinya Nakatani ${ }^{1}$, Kazuya Inoki ${ }^{1}$, Tomoe Tashiro ${ }^{1}$, Sayaka Yamaguchi ${ }^{1}$, Toshihiko Gocho ${ }^{1}$, Fumito Yanagisawa ${ }^{1}$, Kenichi Konda ${ }^{1}$, Masayuki Tojo ${ }^{1}$, Yutaro Kubota ${ }^{1}$, Atsushi Katagiri $^{1}$, Fuyuhiko Yamamura ${ }^{1}$, Yoshiaki Takeuchi ${ }^{1}$, Genshu Tate ${ }^{1}$, Masahiro Komoto ${ }^{1}$, Kimiyasu Yamazaki ${ }^{1}$, Masahiko Murakami ${ }^{2}$, and Hitoshi Yoshida ${ }^{1}$

${ }^{1}$ Showa University

${ }^{2}$ Affiliation not available

November 16, 2020

\begin{abstract}
A 44-year-old man presented to our hospital with lower gastrointestinal bleeding. We performed balloon-assisted enteroscopy, which revealed diverticulum and stricture at the ileum. The patient underwent segmental small bowel resection and diagnosed with Meckel's diverticulum. We should keep in mind the possibility of intestinal stricture due to Meckel's diverticulum.
\end{abstract}

\section{Case Report}

\section{Simultaneous Small Intestinal Bleeding and Stricture Caused by Meckel's Diverticulum}

Shinya Nakatani ${ }^{1}$, Kazuya Inoki ${ }^{1}$, Tomoe Tashiro ${ }^{1}$, Sayaka Yamaguchi $^{1}$, Toshihiko Gocho ${ }^{1}$, Fumito Yanagisawa $^{1}$, Kenichi Konda ${ }^{1}$, Masayuki Tojo ${ }^{1}$, Yutaro Kubota ${ }^{1}$, Atsushi Katagiri ${ }^{1}$, Fuyuhiko Yamamura ${ }^{2}$, Yoshiaki Takeuchi ${ }^{1}$, Genshu Tate ${ }^{3}$, Masahiro Komoto ${ }^{4}$, Kimiyasu Yamazaki ${ }^{4}$, Masahiko Murakami ${ }^{4}$, Hitoshi Yoshida $^{1}$

Institution

1. Department of Medicine, Division of Gastroenterology, Showa University School of Medicine, Tokyo, 142-8666, Japan

2. Endoscopy Center, Showa University Hospital, Tokyo, 142-8555, Japan

3. Department of Pathology, Showa University School of Medicine, Tokyo 142-8555, Japan.

4. Department of Gastroenterological and General Surgery, School of Medicine, Showa University, Tokyo, 142-8555, Japan.

\section{Correspondence to}

Kazuya Inoki, MD, PhD

Department of Medicine, Division of Gastroenterology, Showa University School of Medicine, 1-5-8, Hatanodai, Shinagawaku, Tokyo 142-8666, Japan

Phone: +81-3-3784-8535, Fax: +81-3-3784-7553

E-mail: kinoki@med.showa-u.ac.jp

\section{Keywords}


Meckel's diverticulum, Intestinal stricture, Lower gastrointestinal bleeding, Enteroscopy, Capsule endoscopy

\section{Key Clinical Message}

A 44-year-old man presented to our hospital with lower gastrointestinal bleeding. We performed balloonassisted enteroscopy, which revealed diverticulum and stricture at the ileum . The patient underwent segmental small bowel resection and diagnosed with Meckel's diverticulum. We should keep in mind the possibility of intestinal stricture due to Meckel's diverticulum.

\section{Introduction}

The development of capsule endoscopy (CE) and balloon-assisted enteroscopy (BAE) has enabled endoscopists to better manage obscure gastrointestinal bleeding (OGIB) [1-3]. Among patients who have OGIB, Meckel's diverticulum (MD) can be an important differential diagnosis for OGIB patients. Although MD is usually asymptomatic, it can be a cause of various clinical symptoms such as gastrointestinal (GI) bleeding, intestinal obstruction, and acute abdominal pain. While there are several specific examinations used to diagnose $\mathrm{MD}$, including $\mathrm{CE}$ [4], BAE using a single or double balloon [5], technetium 99m pertechnetate scan [6], and arteriography [7], it is sometimes difficult to obtain a definite diagnosis of MD. The resection of MD is necessary when judged as a source of clinical manifestations.

$\mathrm{CE}$ is less invasive than BAE, and the diagnostic ability of $\mathrm{CE}$ has been reported as superior to BAE for OGIB patients $[2,8]$. Therefore, $\mathrm{CE}$ is usually the first method used to identify OGIB. Although CE is considered as a safe procedure, the risk of capsule retention exists. Capsule retention is defined as the capsule remaining in the GI tract for more than 2 weeks [2]. The main cause of capsule retention is small intestinal stricture. The reported etiology of small intestinal stricture includes drug-induced small intestinal stricture due to nonsteroidal anti-inflammatory drugs, tumors, inflammatory bowel disease, postoperative stricture, and stricture induced by radiation [9]. Here, we report a case of simultaneous small intestinal bleeding and stricture due to MD.

\section{Case report}

A 44-year-old man with a history of occasional melena since childhood presented to our hospital with a complaint of acute lower GI bleeding. The physical examination showed no abnormal findings. Laboratory evaluation revealed decreased hemoglobin $(10.4 \mathrm{mg} / \mathrm{dl})$. The patient underwent esophagogastroduodenoscopy and total colonoscopy. Although bright red blood was seen with colonoscopy, the source of bleeding was not identified (Figure 1a, 1b). Furthermore, the source of bleeding was not found with abdominal contrast-enhanced computed tomography (CT). The patient was diagnosed with OGIB, and CE was performed subsequently to examine the small intestine. Normal intestinal fluid was shown by CE, and it was difficult to identify the source of bleeding (Figure 2a, 2b). We explained the necessity of performing BAE to the patient, however he did not consent to BAE at that time. Fortunately, the bleeding stopped spontaneously. Magnetic resonance imaging in the outpatient department at follow-up did not identify the source of bleeding. The patient was prescribed with Rebamipide at the time of follow-up. After 9 months, the patient presented to our hospital again with lower GI bleeding. Since detailed testing including CE had already been performed with the previous bleeding, BAE was the first test performed after obtaining informed consent. A diverticulum was revealed by BAE in the ileum at the $50 \mathrm{~cm}$ oral side from the ileocecal valve, and intestinal stricture was observed near the diverticulum (Figure 3a). It was difficult to traverse the colonoscope, and the small bowel series with Gastrografin showed the blind end that corresponds to the diverticulum and the stricture (Figure $3 \mathrm{~b})$. Although no active bleeding was observed during the BAE procedure, there were no other possible sources of bleeding. Therefore, we diagnosed MD as the source of bleeding, and decided to treat the patient surgically. The diverticulum was apparent, and segmental small bowel resection with primary anastomosis was performed (Figure 4a,4b). Pathology of the surgical specimen showed the true diverticulum with a small erosion (Figure 4c), and heterotopic gastric mucosa was seen in the diverticulum; therefore, MD was diagnosed pathologically (Figure 4d). The patient has had no lower GI bleeding since surgery.

\section{Discussion}


We encountered an OGIB patient who eventually experienced small intestinal stricture due to MD. The clinical course of this patient suggests two important clinical issues. First, although the frequency is low, OGIB patients may have small intestinal strictures due to inflammation from MD. Second, the diagnosis of bleeding due to MD is still sometimes difficult to determine, even when using several diagnostic modalities.

In the present case, intestinal stricture due to MD was diagnosed by BAE after the second occurrence of hematochezia. Although capsule retention was avoided with this patient, transient capsule retention inside the MD has been reported in other cases [10]. A possible explanation of the clinical course of the present case was that the bleeding from the ulcer in the ileum occurred; thereafter, stricture developed as a result of scar formation after ulcer healing. The ulcer formed due to exposure to acid secretion from the heterotopic gastric mucosa of MD. Because we performed CE after the second occurrence of hematochezia, there was a possibility that capsule retention might occur since the stricture was too severe to traverse with an enteroscope. More commonly, capsule retention due to drug-induced stricture from non-steroidal anti-inflammatory drugs has been reported, usually presenting as diaphragmatic stricture $[11,12]$. Rezapour $\mathrm{M}$ et al. conducted a metaanalysis of retention associated with $\mathrm{CE}$ and reported that capsule retention occurred in $2.1 \%$ of patients with OGIB [9]. Furthermore, $3 \%$ of patients with capsule retention in that meta-analysis developed obstructive symptoms. Patency CE is used for patients who have a high risk of intestinal stricture, and reports indicate that it has reduced the risk of capsule retention by half with IBD patients [9]. Performing patency CE for all OGIB patients is not recommended, considering the low incidence of stricture due to MD (such as our case), but endoscopists should keep in mind the possibility of stricture due to MD when examining OGIB. If the possibility of MD is already high with other modalities, patency $\mathrm{CE}$ may be an option before CE.

It was difficult to diagnose MD before surgery with the present case. Several specific diagnostic tools have been reported to help diagnose $\mathrm{MD}$, including arteriography, technetium $99 \mathrm{~m}$ pertechnetate scan, CE, and BAE. Among these, arteriography and technetium $99 \mathrm{~m}$ pertechnetate scan are regarded as the standard tools to diagnose MD. If bleeding is heavy, arteriography can be a good option. In patients who have less bleeding, high-resolution CT arteriography can be a good option, and its overall sensitivity for detecting active acute GI bleeding is reported to be as high as $85.2 \%$ [13]. If bleeding stops spontaneously, it could be difficult to detect the source of bleeding with high-resolution CT arteriography. The technetium $99 \mathrm{~m}$ pertechnetate scan failed to diagnose the presence of MD with the present case, although the heterotopic gastric mucosa was confirmed pathologically after surgery. The sensitivity of the technetium $99 \mathrm{~m}$ pertechnetate scan for adult MD has been reported to be approximately 60\% [6]. Krstic SN et al. reported that CE had a high positive predictive value of $84.6 \%$ for the diagnosis of MD [4]. However, in the same study, CE identified the source of bleeding in only $44.6 \%$ of OGIB patients. In the present case, several diagnostic tools, including high-resolution CT arteriography, technetium 99m pertechnetate scan, and CE, failed to diagnose MD. Considering the insufficient sensitivity of some tests to diagnose MD, several examinations, including BAE, should be carried out to diagnose MD. Although BAE is an invasive procedure, it could be sometimes a preferable option compared with other modalities, especially for the diagnosis of MD $[5,14]$.

In addition to the difficulty of MD diagnosis, we should keep in mind the possibility of intestinal stricture due to MD when seeing OGIB patients.

\section{Acknowledgments}

We would like to thank Editage (www.editage.com) for English language editing.

\section{Author contribution}

All authors reviewed the manuscript.

Prof. Masahiko murakami, Dr. Kimiyasu yamazaki and Dr. Masahiro Komoto provided all the details about the surgical treatment.

Dr Genshu Tate described the histopathological features and provided the pictures of the biopsies.

\section{Confrict of Interest}


None declared.

CONFLICT OF INTEREST

None declared

CONFLICT OF INTEREST

None declared

CONFLICT OF INTEREST

None declare

\section{References}

Raju GS, Gerson L, Das A, et al. 2007. American Gastroenterological Association (AGA)

Institute medical position statement on obscure gastrointestinal bleeding. Gastroenterology 133:1694-1696.

2. Liao Z, Gao R, Xu C, et al. 2010.Indications and detection, completion, and retention rates of small-bowel capsule endoscopy: a systematic review. Gastrointest Endosc 71:280-286.

3. Shinozaki S, Yamamoto H, Yano T, et al. 2010.Long-term outcome of patients with obscure gastrointestinal bleeding investigated by double-balloon endoscopy. Clin Gastroenterol Hepatol 8:151-158.

4. Krstic SN, Martinov JB, Sokic-Milutinovic AD, et al.2016.Capsule endoscopy is useful diagnostic tool for diagnosing Meckel's diverticulum. Eur J Gastroenterol Hepatol 28:702-707.

5. Fukushima M, Kawanami C, Inoue S, et al. 2014.A case series of Meckel's diverticulum: usefulness of double-balloon enteroscopy for diagnosis. BMC Gastroenterol 14:155.

6. Lin S, Suhocki PV, Ludwig KA, et al. 2002 Gastrointestinal bleeding in adult patients with Meckel's diverticulum: the role of technetium 99m pertechnetate scan. South Med J 95:1338-1341.

7. Routh WD, Lawdahl RB, Lund E, et al.1990.Meckel's diverticula: angiographic diagnosis in patients with non-acute hemorrhage and negative scintigraphy. Pediatr Radiol 20:152-156.

8. Adler DG, Knipschield M and Gostout C. 2004. A prospective comparison of capsule endoscopy and push enteroscopy in patients with GI bleeding of obscure origin. Gastrointest Endosc 59:492-498.

9. Rezapour M, Amadi C and Gerson LB. 2017.Retention associated with video capsule endoscopy: systematic review and meta-analysis. Gastrointest Endosc 85:1157-1168.e1152.

10. Courcoutsakis N, Pitiakoudis M, Mimidis K, et al. 2011. Capsule retention in a giant Meckel's diverticulum containing multiple enteroliths. Endoscopy 43 (Suppl):E308-309.

11. Ben Soussan E, Antonietti M, Hervé S, et al.2004. Diagnostic yield and therapeutic implications of capsule endoscopy in obscure gastrointestinal bleeding. Gastroenterol Clin Biol 28:1068-1073.

12. Sears DM, Avots-Avotins A, Culp K, et al.2004. Frequency and clinical outcome of capsule retention during capsule endoscopy for GI bleeding of obscure origin. Gastrointest Endosc 60:822-827.

13. García-Blázquez V, Vicente-Bártulos A, Olavarria-Delgado A, et al. 2013. Accuracy of CT angiography in the diagnosis of acute gastrointestinal bleeding: systematic review and meta-analysis. Eur Radiol 23:11811190.

14. Hong SN, Jang HJ, Ye BD, et al. 2016. Diagnosis of Bleeding Meckel's Diverticulum in Adults. PLoS One 11:e0162615.

\section{Figure Legends}

Figure 1. Colonoscopy revealed bright red blood at the (a) colon and (b) ileum. 
Figure 2. (a) (b) Capsule endoscopy showed no evidence of intestinal bleeding.

Figure 3. Endoscopic images obtained by BAE showing (a) the diverticulum (arrow) and the intestinal stricture (arrowhead) near the diverticulum (b). Small bowel series during BAE showing diverticulum (arrow) and small intestine beyond the stricture (arrowhead).

BAE: balloon-assisted enteroscopy

Figure 4. Intraoperative photo showing (a) the diverticulum in the ileum; (b) gross specimen of resected small intestine including the diverticulum. Pathology of the surgical specimen showing (c) the true diverticulum with erosion (arrow) with HE staining. The magnifying image of the yellow square in (c) showing (d) the ectopic gastric mucosa with HE staining.

HE: hematoxylin and eosin

\section{Figure 1}

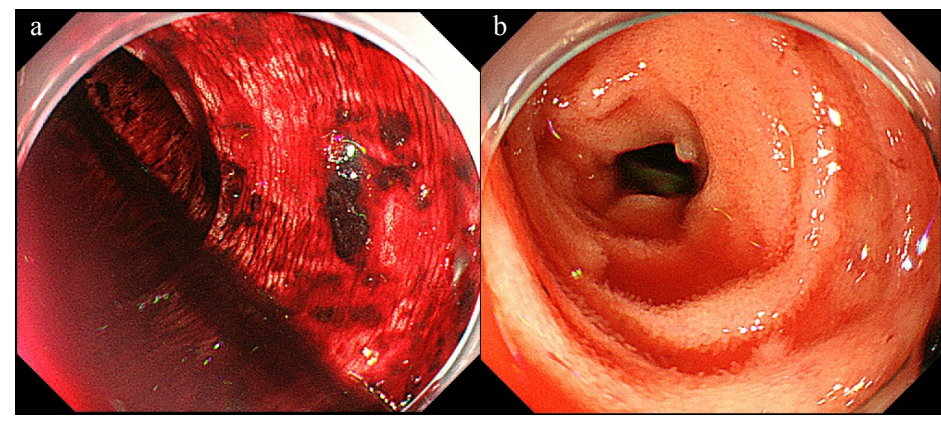

\section{Figure 2}

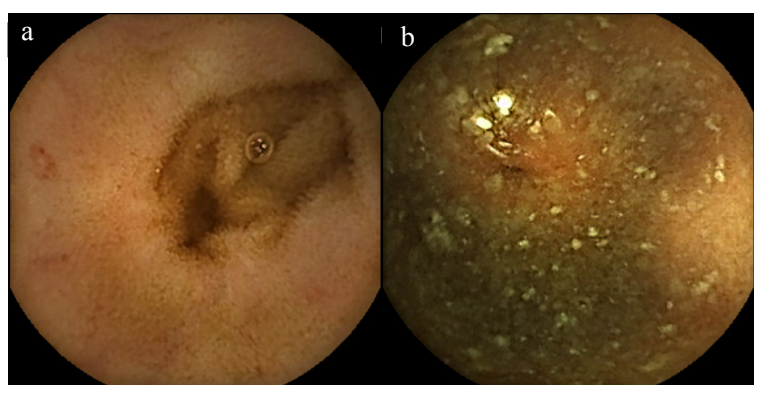


Figure 3

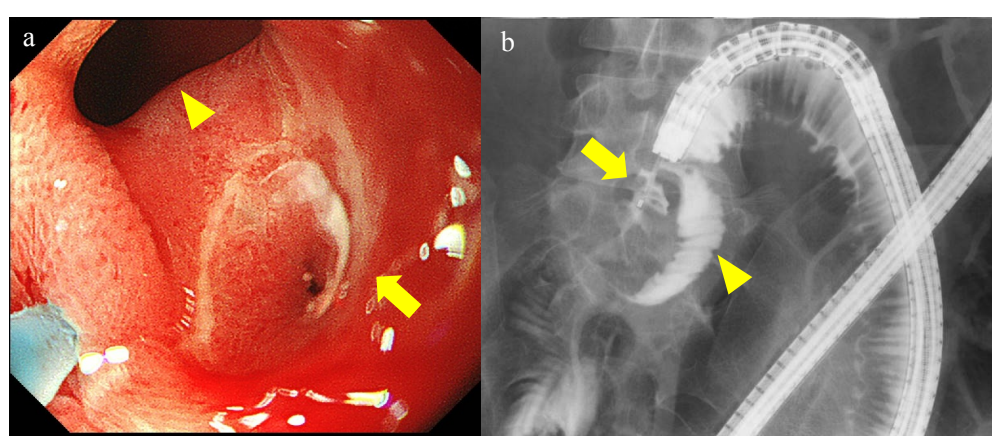

Figure 4

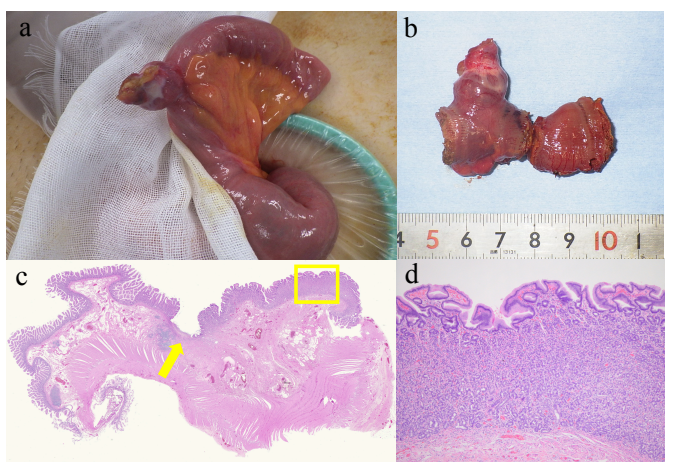

